\title{
Cognitive Behavior Modeling of Manual Rendezvous and Docking Based on the ACT-R Cognitive Architecture*
}

\author{
Chunhui Wang ${ }^{2}$, Yu Tian², Yanfei Liu ${ }^{1}$, Shanguang Chen ${ }^{2}$, Zhiqiang Tian², \\ and Junsong $\mathrm{Li}^{1}$ \\ ${ }^{1}$ Zhejiang Sci-Tech University, Hangzhou, 310018, China \\ ${ }^{2}$ National Key Laboratory of Human Factors Engineering, Astronaut Research and Training \\ Center of China, Beijing, China 100094 \\ chunhui_89@yahoo.com.cn, cctian@126.com, yliu@zju.edu.cn, \\ tianzhiqiang2000@163.com, 1jscg@sina.com
}

\begin{abstract}
Astronauts are exposed to numerous stressors during spaceflights, to reduce the faulty operations and improve human performance in manually controlled rendezvous and docking (manual RVD) of space vehicles, a method, which applies sophisticated cognitive architecture Adaptive control of thought-rational (ACT-R ) to model astronaut's cognitive behaviors and investigate the cognitive components influencing human performance, is proposed in this paper. To model the cognitive behaviors in completing manual RVD task, the declarative knowledge of the cognitive processes was obtained from experiments, the procedural knowledge was acquired by analyzing the relationship between cognitive processes and behaviors, and the model parameters were set up according to the boundary conditions and task characteristics. Manual RVD cognitive behavior model is then built up based on the declarative knowledge, the procedural knowledge and the model parameters. Comparisons of model running results and manual control results were performed to test the model's validation. ACT-R control panel tool was also used to analyze the process of model's running and manual control behaviors. Results of the comparisons indicate that the ACT-R model proposed in this paper is in accordance with human cognitive behaviors.
\end{abstract}

Keywords: ACT-R, manually controlled Rendezvous and docking task, cognitive behavior modeling, model's validation.

\section{Introduction}

Cognitive models are appearing in all fields of cognition at a rapidly increasing rate, ranging from perception to memory to problem solving and decision-making. A large

\footnotetext{
* This work is supported by 973 Program of China under Grant No. 2011CB711000, Zhejiang Provincial Natural Science Foundation under Grant No. LY12C09005, Y1110477, and National Natural Science Foundation of China under Grant No.61100183.

*** Corresponding author.
} 
percent of the articles appearing in major theoretical journals of Cognitive Science involve cognitive modeling. Furthermore, applications of cognitive modeling are beginning to spill over into other fields including human factors, clinical psychology, cognitive neuroscience, agent based modeling in economics, and many more [1]. As an effective research method for human's cognition, cognitive modeling is well accepted and widely studied recently, such as Anderson and Fincham(2012) track problem-solving using brain imaging[2], Janssen and Gray(2012) Reinforce Learning based Models of Cognition[3], Kennedy and Ritter(2012) address the topics related to cognitive modeling of beyond-rational cognition[4], Gunzelmann (2011) elaborate a variety of disciplines and methodologies on Modeling Spatial Cognition[5], and Borst and Taatgen (2011) perform model-based fMRI analyses by using high-level symbolic cognitive models to enable fine-grained exploratory fMRI research[6] etc. Thus cognitive modeling is becoming an essential tool for Cognitive Science in particular and the Social Sciences in general.

As one of the most typical and widely used cognitive architecture and a unified theory of cognition [7], ACT-R aims at using software to simulate a full range of cognitive tasks, has been used to construct models to simulate human's task performing and cognitive process, such as Beckmann and Yilmaz(2012) introduce a realization of a more complicated interactive warning framework to avoid operator mistakes during maintenance[8], Kennedy and Patterson (2102) present an ACT-R model to predict the development of intuitive decision-making in humans[9] etc. In addition, ACT-R has been used to access and improve human's performance in complex and adaptive system such as aircraft operations, nuclear power operations and supervisory control. Gunzelmann and Moore (2012) tries to account for the function of a particular brain area and raises questions for understanding cognitive performance [10], Wayne and David discuss how to use cognitive modeling to access human(pilot, computer user, and car driver) performance[11-12], and Gunzelmann et .al use cognitive modeling to study impaired performance due to sleep deprivation [13-14].

Manual RVD is a complex and difficult task for astronauts. During manual RVD, due to human's capability in observation, analysis, judgment, decision-making and handling, the astronauts can make judgment and decision timely for the system's fault and can improve probability of success for manual RVD task. However, Astronauts are exposed to numerous stressors during spaceflights, such as microgravity, confinement, and radiation, all of which may impair human cognitive capabilities. In some critical operations for spaceflight, such as operating the mechanical arms, extravehicular activities, and driving the spacecraft, etc., fault operation may cause serious disasters. Moreover, due to limitations for test conditions, uncertainty and poor features of experimental results in the study of human cognitive behavior for spaceflight, experimental researches are difficult to implement in reality. As a cognitive modeling tools, The ACT-R provide studies helps for astronauts' performance on orbit. 


\section{Data Acquisition and Knowledge Extraction}

\subsection{Data Acquisition Experiment}

To obtain the knowledge and model parameter for manual RVD task's cognitive behavior model, manual RVD experiment is designed and implemented to collect the raw data. For convenience, the experiment only consider the situation that manual RVD operation start from distance 20 meters of two space vehicles to the manual RVD task being finished, longitudinal speed maintains $2 \mathrm{~m} / \mathrm{s}$ and remains unchangeable, the initial displacement in the horizontal and vertical direction of two vehicles are both 2 meters, there is no initial velocity in the direction of horizon and vertical, and there is no posture (rotation, pitch and yaw) operation during the whole manual RVD task. That is to say that there is only three dimensions' moving operation being considered in the designed experiment.

During experiment of operator's manual RVD task, the operator's behavior and vehicle's status are recorded. Table 1 shows the snippet of the recorded information as examples.

Table 1. Cognitive behavior for manual RVD task

\begin{tabular}{|c|c|c|c|c|c|c|c|}
\hline \multirow{2}{*}{$\begin{array}{c}\text { Distance / } \\
\text { Time }\end{array}$} & \multirow{2}{*}{$\begin{array}{c}\text { Vision } \\
\text { Vehicle's } \\
\text { status } \\
\end{array}$} & \multicolumn{4}{|c|}{ Information processing } & \multicolumn{2}{|c|}{ Manual control } \\
\hline & & Attention & Memory & Judgment & $\begin{array}{c}\text { Decision } \\
\text { making }\end{array}$ & $\begin{array}{c}\text { Move } \\
\text { op. }\end{array}$ & Time elapse \\
\hline 0’0”(20m) & Q1, N & RU & Lo, Po & RU & $\mathrm{O}: \mathrm{S}$ & NULL & \\
\hline 0'0"-0'1"' & $\mathrm{Q} 1, \mathrm{R}$ & RU & Lo,Po & RU & $\mathrm{O}: \mathrm{L}$ & $\mathrm{L}, 1$ & $0.5^{\prime}$ \\
\hline$\ldots$ & & & & & & & \\
\hline
\end{tabular}

In Table 1, Q1 - Quadrant I, N - No motion trend, RU-Right upper corner location, O-Operation, R-Right (in Vehicle status column represent right moving, and in Decision making column and Move op. column represent for right operation), LoLocation, Po - Posture, S -Start operation, L - Left, and 1 - 1 time.

\subsection{Knowledge Extraction and Model's Parameters}

The most important thing in ACT-R is extraction for declarative knowledge, procedural knowledge and model's parameter. The declarative knowledge is some conceptions definition for the task or some facts, such as the operations, the vehicle's status and the relationship between drone's size and vehicle's distance etc. The procedural knowledge is rules for decision-making of the model, and procedural knowledge is acquired by analyzing the relationship between cognitive processes and behaviors. Typical procedural knowledge such as determining operation behavior according the vehicle's location or determining the vehicle's distance according to the drone's size can be extracted from the experimental data recorded in Table 1. The model parameters are set up according to the boundary conditions, some custom constants predefinition and the task's characteristics, such as the vehicle's maximum speed, maximum contact speed and the maximum allowable deviations etc. 


\subsection{Manual RVD Cognitive Behavior Modeling}

The cognitive model on ACT-R architecture platform is programed in lisp programming language and composed of two main parts, the first part is the manual controlling part for user's manual controlling GUI, and another part is the model part. The manual controlling part implement the functions for dealing of user's operation input, vehicle's movement and related information display in GUI. The model part includes the chunk type's definition, declarative knowledge, first goal of the model and all the procedural knowledge.

\section{Model's Running and Validation}

The model can be run either in manual controlling or automatic mode. As in the manual controlling mode, the operator implement manual RVD task by controlling the vehicle's movement according the vehicle's moving status, and vehicle's running status and operations are recorded by model. When model running in automatic mode, the model start running according to model's first goal, and then it fires productions circularly along with the model's new goal. The model will end as there is no any production fit the goal of the goal buffer and being trigged.

The comparison method for model running results and manual control process is used to test the model's validation, and also ACT-R control panel tool is used to analysis the process of model's running and manual control behavior. Figure 1 show the time period comparison for manual operation result and model's running result.

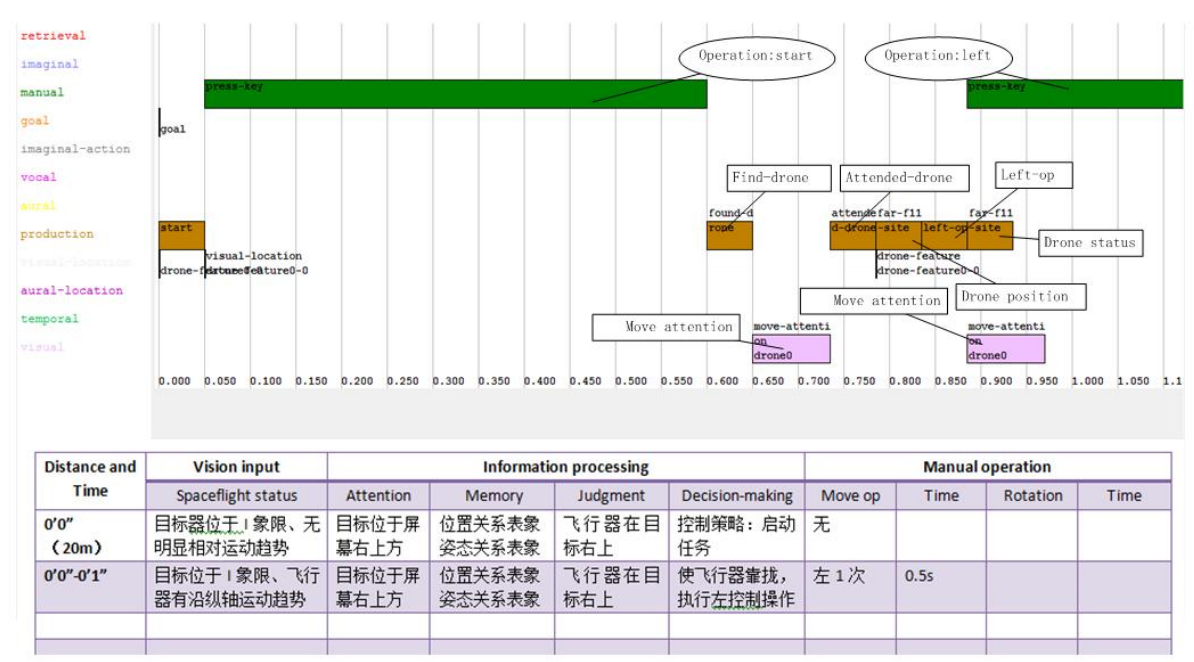

Fig. 1. Behavior Comparison results for manual operation and model's running

The upper half section of the figure 1 is the cognitive procedure when model running and displayed in ACT-R control panel tool. On the left of this section we see the names of all the buffers and along the bottom we see the time. For each buffer 
there are boxes displayed which correspond to the actions which occurred related to that buffer. The boxes in the production row show the names of the productions which fired, but for the other buffers they display the chunk-type of the request which was made at the top of the box and the name of the resulting chunk (if there was one) along the bottom. The lower half part is the operator's behavior procedure as the manual RVD experiment being conducted. From the figure we can find the model's results are is quite up to the manual control operations.

The comparison of vehicle location for manual control and model's automatic running in the procedure of manual RVD tasks are implemented. Fig. 2 shows the comparison results for the horizontal and vertical direction.

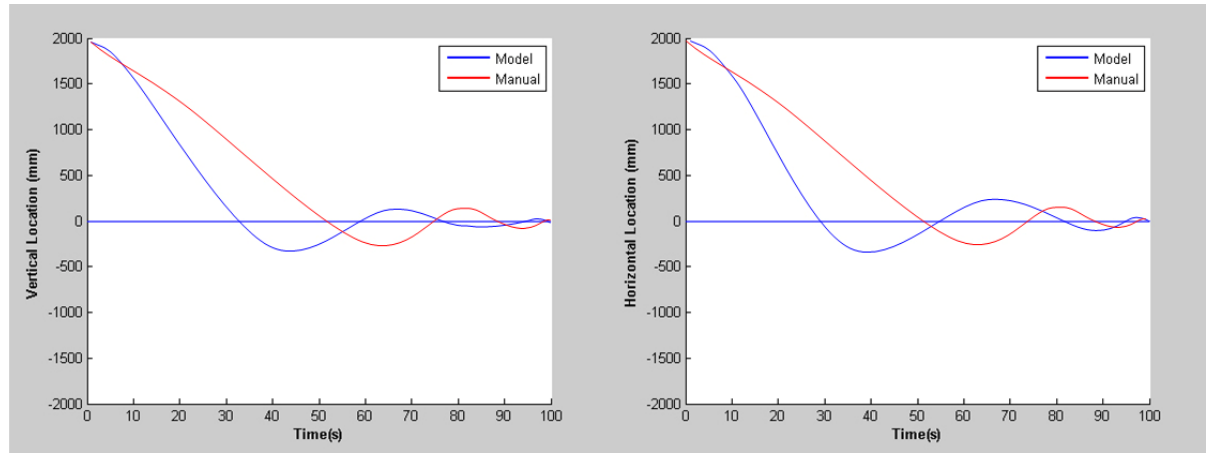

Fig. 2. The location's comparison results for manual operation and model's running

The figures show that the model's running procedure is in accordance with the moving trend of manual control procedure in manual RVD task.

\section{Discussion}

To get the model to fit the manual cognitive behavior requires not only writing a plausible set of productions which can accomplish the task, but also setting the ACT$\mathrm{R}$ parameters that control the behavior as described in the equations governing the operation of declarative memory. This paper only describes the model running result with the default values for the parameters and hence the model's result shows some of the general trends in figure 2. There are lots of parameters in ACT-R that reflects human's characteristic such as base-level-activation, retrieve threshold and latency factor, etc. By adjusting the model's parameter, the manual RVD task's performance is not investigated here.

\section{Conclusion}

ACT-R is a developing theory and architecture, on the one hand it is complex both in theory and architecture, on the other hand it has been developed very quickly recently. Cognitive model of a simple task is developed in this paper and lots of simplifications have been made. There is much work to do in developing models for sophisticated 
manual RVD tasks and assessing difference behavioral performance with parameter's tuning in the model.

The contribution of this paper is that a method for analyzing the human cognitive behavior is proposed. Comparisons of both the cognition procedures and vehicle's locations were performed to validate the cognitive model.The analyzing result shows that the model based on the ACT-R cognitive architecture is effective in modeling astronaut's cognitive behavior in the manual RVD task.

\section{References}

1. Busemeyer, J.R., Diederich, A.: Cognitive modeling, Pages 1. Sage Publications (2009) (incorporated)

2. Anderson, J.R., Fincham, J.M., Schneider, D.W., Yang, J.: Using brain imaging to track problem-solving in a complex state space. NeuroImage 60, 633-643 (2012)

3. Janssen, C.P., Gray, W.D.: When, What, and How Much to Reward in Reinforcement Learning based Models of Cognition. Cognitive Science 36(2), 333-358 (2012)

4. Kennedy, W.G., Ritter, F.E., Juvina, I., Gratch, J., Young, R.M.: ICCM Symposium on Cognitive Modeling of Processes "Beyond Rational". In: Proceedings of the 11th International Conference on Cognitive Modeling, Berlin, Germany, April 12-15 (2012)

5. Gunzelmann, G.: Introduction to the Topic on Modeling Spatial Cognition. Topics in Cognitive Science 3(4), 628-631 (2011)

6. Borst, J.P., Taatgen, N.A., Van Rijn, H.: Using a Symbolic Process Model as input for Model-Based fMRI Analysis: Locating the Neural Correlates of Problem State Replacements. NeuroImage 58(1), 137-147 (2011)

7. Beckmann, M., Yilmaz, U., Pöhler, G., Wegerich, A.: A framework for task accomplishment using an ACT-R simulation. In: Proceedings of the 11th International Conference on Cognitive Modeling. Universitaetsverlag der TU Berlin, Berlin (2012)

8. Kennedy, W.G., Patterson, R.E.: Modeling Intuitive Decision Making in ACT-R. In: Proceedings of the 11th International Conference on Cognitive Modeling, Berlin, Germany, April 12-15 (2012)

9. Gunzelmann, G., Moore, L.R.: Evaluating the relationship between neuropsychological function and cognitive performance. In: Proceedings of the Thirty-Fourth Annual Meeting of the Cognitive Science Society, pp. 414-419 (2012)

10. Wayne, G., David, K., Guk-Ho, G., Sang-Hwan, K., Ben-Asher, N., Joachim, M., Shi, C., Yili, L., Cleotilde, G., Glenn, G., Kevin, G.: Symposium on Human Performance Modeling. In: Proceedings of the 11th International Conference on Cognitive Modeling, Berlin, Germany, pp. 324-327 (April 2012)

11. Schoelles, M., Gray, W.D.: SimPilot: An exploration of modeling a highly interactive task with delayed feedback in a multitasking environment. In: Proceedings of the 11th International Conference on Cognitive Modeling, Berlin, Germany, vol. 66 (2012)

12. Gunzelmann, G., Gluck, K.A., Moore, L.R., Dinges, D.F.: Impaired performance due to sleep deprivation: A role for diminished knowledge access. Cognitive Systems Research 13(1), 1-11 (2012)

13. Gunzelmann, G., Moore, L.R., Salvucci, D.D., Gluck, K.A.: Sleep loss and driver performance: Quantitative predictions with zero free parameters. Cognitive Systems Research 12(2), 154-163 (2011)

14. Anderson, J.R., Bothell, D., Byrne, M.D., Douglass, S., Lebiere, C., Qin, Y.: An integrated theory of the mind. Psychological Review 111(4), 1036-1060 (2004) 